\title{
Hyperthermic intraperitoneal chemotherapy with oxaliplatin as treatment for peritoneal carcinomatosis arising from the appendix and pseudomyxoma peritonei: a survival analysis
}

Eric Marcotte ${ }^{1}$, Pierre Dubé ${ }^{2}$, Pierre Drolet ${ }^{2}$, Andrew Mitchell ${ }^{3}$, Suzanne Frenette ${ }^{4}$, Guy Leblanc ${ }^{1}$, Yves E Leclerc ${ }^{1}$ and Lucas Sideris ${ }^{1 *}$

\begin{abstract}
Background: Appendiceal peritoneal carcinomatosis (PC) is rare and its long-term prognosis is poor. The aim of this study was to evaluate the results of an aggressive treatment approach used in our institution for the last eight years.

Methods: Data from all patients with PC arising from the appendix were prospectively collected and analyzed. Treatment consisted of complete surgical cytoreduction (CRS), followed by hyperthermic intraperitoneal chemotherapy (HIPEC) with oxaliplatin $\left(460 \mathrm{mg} / \mathrm{m}^{2}\right)$ at $43^{\circ} \mathrm{C}$ over 30 minutes. Ronnett's histologic classification was used for tumor grading.

Results: Between February 2003 and April 2011, 78 patients underwent laparotomy with curative intent. The mean follow-up period was 33.7 months. A total of 58 patients received HIPEC, but 11 patients could not have CRS and received no HIPEC. Nine patients with a negative second-look surgery also received no HIPEC. The five-year overall survival for the entire cohort was 66.2\%; 100\% for the negative second-look patients, $77 \%$ for the HIPEC patients and $9 \%$ for the unresectable patients $(P<0.0001)$. A total of 15 patients $(25.9 \%)$ had isolated peritoneal recurrence, no patient had visceral recurrence only, and five patients (8.6\%) had both. In regards to the five-year disease-free survival for the HIPEC patients, histologic grade (disseminated peritoneal adenomucinosis 100\%, peritoneal mucinous carcinomatosis with intermediate features $40 \%$, peritoneal mucinous carcinomatosis $20 \% ; p=0.0016$ ) and completeness of cytoreduction (CCR-0 56\%, CCR-1 24\%; $P=0.0172$ ) were prognostic factors. There was one postoperative mortality. The major complication rate for patients treated with HIPEC was 40\%, including intra-abdominal abcess (17\%), hemorrhage (12\%) and anastomotic leak (10\%). One patient in the HIPEC group experienced temporary grade II neuropathy and grade III thrombocytopenia.
\end{abstract}

Conclusions: This therapeutic approach seems both feasible and safe in selected patients. Recurrence is, however, frequent and represents a challenge.

Keywords: Peritoneal neoplasms, Appendiceal neoplasms, Hyperthermic intraperitoneal chemotherapy, Oxaliplatin

\footnotetext{
* Correspondence: lucas.sideris@umontreal.ca

'Department of Surgery, Hôpital Maisonneuve-Rosemont, University of Montreal, 5415 boulevard de l'Assomption, Montréal, Québec H1T 2 M4,

Canada

Full list of author information is available at the end of the article
} 


\section{Background}

Neoplasms of the appendix are rare, with their ageadjusted incidence rate being 0.12 to 2 cases per 1,000,000 people per year [1]. They are found in about $1 \%$ of appendectomy specimens [2]. Epithelial tumors, which represent the majority of the appendiceal cancers [1], often present with peritoneal dissemination.

For those cases, the traditional approach has consisted in repetitive debulking surgery and the long-term prognosis (five and ten-year overall survival (OS)) of these patients has been demonstrated in two studies as being 53\% and $32 \%$ [3], and $65 \%$ and $21 \%$ [4], respectively. A more aggressive approach consisting of complete surgical cytoreduction (CRS) and intra-peritoneal chemotherapy has been introduced by Gonzalez-Moreno and Sugarbaker in 1983, with five- and ten-year OS rates of $71.9 \%$ and $54.5 \%$, respectively [5]. There are many studies published in the last decade similarly showing improvement in long-term survival when compared to traditional debulking surgeries, with five- and ten-year OS ranging from 40 to $87 \%$ and 50 to $74 \%$, respectively [6-15]. The addition of hyperthermia to chemotherapeutic agents has been shown to enhance their cytotoxicity [16] and their penetration into tumors [16-18].

There is great variability in regards to the agent used for the intraperitoneal chemotherapy protocol. Some investigators use mitomycin-C or 5-fluorouracil, whereas Elias et al. have introduced oxaliplatin as a hyperthermic intraperitoneal chemotherapy (HIPEC) agent for peritoneal carcinomatosis (PC) arising from the appendix [19]. In a phase one study, intraperitoneal oxaliplatin had an advantageous pharmacokinetic profile, displaying very high intraperitoneal concentrations but low systemic toxicity because of limited systemic absorption [20]. Its advantages also include a reduced duration of perfusion, from 90 minutes for mitomycin-C to 30 minutes, as well as a potentialization of peritoneal concentration, while reducing systemic (plasmatic of portal) concentrations in the context of HIPEC, as shown through animal studies performed in our center [18].

The aim of this study was to analyze overall surgical outcome, long-term survival, as well as to identify factors of prognostic value for the patients treated in our institution over the last eight years. It is an update on our series published in 2008 and remains the first to use oxaliplatin exclusively as a cytotoxic agent in HIPEC for PC arising from the appendix.

\section{Methods}

\section{Patients}

From a prospective database, we included all patients $(\mathrm{n}=78)$ with peritoneal surface dissemination of epithelial appendiceal tumors treated in our center between February 2003 and February 2011. Our institution's clinical trial review board approved this study. Hôpital MaisonneuveRosemont, University of Montreal. Patients are agreeing to be part of the database when consenting to care. The following factors were analyzed: demographic data, surgical procedures, pathologic diagnosis, complications and length of hospital stay.

Patients were offered treatment consisting of maximal surgical cytoreduction and HIPEC with oxaliplatin if they fulfilled the following criteria: diagnosis proven by histological examination, no evidence of visceral metastasis on computed tomographic (CT) imaging of the chest and abdomen, a technically resectable disease, and a general health status good enough to tolerate the proposed surgery.

\section{Surgery for peritoneal carcinomatosis and hyperthermic intraperitoneal chemotherapy}

The surgical technique for both $\mathrm{PC}$ and open-abdomen HIPEC with oxaliplatin $\left(460 \mathrm{mg} / \mathrm{m}^{2}\right.$ at 42 to $44^{\circ} \mathrm{C}$ for 30 minutes) has been previously described [21].

\section{Postoperative course}

Patients were followed up on every day following surgery. Surgical complications were graded using a five-point scale [22]. Minor complications (grade I or II) that were managed with pharmacological treatment (for example urinary tract infection treated with antibiotics) or non-invasive procedures (for example nasogastric tube for postoperative ileus) were not considered. Major complications were defined as grade III to V. A grade III complication is one requiring surgical, endoscopic or radiological intervention (such as an intra-abdominal abscess). A grade IV complication is considered life-threatening and requires ICU management (such as hemorrhagic shock). Grade $\mathrm{V}$ is defined as postoperative mortality. We included all complications directly related to the surgical procedure, even if they occurred beyond 30 days postoperatively. Chemotherapy-associated complications were graded according to the Common Terminology Criteria for Adverse Events (CTCAE) version 4.0.

\section{Pathology}

Pathologic classification was performed by an experienced pathologist in our hospital. When surgery of the primary tumor had not been performed in our institution, the pathology material was reviewed by the same pathologist to provide uniform application of diagnostic and grading criteria. Tumor grading of both primary (when available) and peritoneal deposits was done according to Ronnett's histologic classification [23]. Disseminated peritoneal adenomucinosis (DPAM) was characterized histologically by the presence of scant low-grade adenomatous mucinous epithelium within abundant extracellular mucin and associated fibrosis. Peritoneal mucinous carcinomatosis (PMCA) displayed the cytologic and architectural features of highergrade mucinous carcinoma associated with extracellular mucin, often with invasive components and sometimes 
demonstrated signet-ring cell differentiation. Peritoneal mucinous carcinomatosis with intermediate features (PMCA-I) presented with combined DPAM and PMCA characteristics; such tumors were invariably derived from well-differentiated mucinous adenocarcinomas of the appendix.

\section{Systemic chemotherapy}

Perioperative systemic chemotherapy was administered to all PMCA patients. Systemic chemotherapy was also administered preoperatively for three to six months to patients with lower grade disease with extensive disease, defined as an estimated Peritoneal Cancer Index (PCI) [24] greater than 25. This systemic treatment was aimed at diminishing the tumor burden in order to maximize the chance of complete surgical cytoreduction thereafter. Chemotherapy consisted of 5-fluorouracil with irinotecan or oxaliplatin.

\section{Follow-up}

Patients were seen at an outpatient clinic at four-month intervals, at which a physical examination was performed. A CT scan of the abdomen and pelvis was performed every four months for two years, every six months for an additional three years and yearly thereafter.

\section{Statistics}

Data were obtained from a prospective database of clinical records as well as surgical, pathological and radiological reports. No patients were lost during the follow-up period. Kaplan-Meier's survival curves were established and were compared with log-rank tests. The Cox proportionalhazards regression model was used to analyze the influence of different factors on disease-free survival (DFS) and OS. Differences were considered significant at $P \leq 0.05$. Data were analyzed using Prism (GraphPad Software, San Diego, California, United States) and JMP ${ }^{\mathrm{rm}}$ 6.0 (SAS Institute Inc. Cary, North Carolina, United States).

\section{Results}

Between February 2003 and February 2011, 78 patients with a PC originating from the appendix underwent laparotomy with curative intent. There were 34 males and 44 females, with a mean age of 50 (range 32 to 70 ) years. The primary tumor had been removed in 72 patients. At laparotomy, 14 patients were found to have unresectable disease, either because of too extensive PC or intraoperative discovery of visceral metastasis. Of note, two of these patients underwent repeat surgery with successful cytoreduction and HIPEC after six months of systemic chemotherapy. Nine other patients initially diagnosed with a mucinous tumor of the appendix with limited peritoneal disease had no evidence of PC when a second-look laparotomy was performed in our center six to twelve months later. All these patients had undergone appendectomy along with the complete removal of peritoneal tumor in another center before being referred to us. Four of these nine patients with well-differentiated cystadenocarcinoma of the appendix underwent complementary right hemicolectomy at second-look surgery for staging purposes. Since these patients had no evidence of residual disease they were not treated with HIPEC.

Complete surgical cytoreduction followed by HIPEC was performed in 58 patients. The CCR Completeness of Cytoreduction score [25] was 0 for 43 patients and one for 15 patients. In the HIPEC group, the median peritoneal cancer index was 13 (2 to 28), patients had a mean of 1.4 (0 to 5 ) organs resected and 0.6 (0 to 2$)$ anastomosis. Median operative time was 362 (135 to 855$)$ minutes and the median blood loss was 600 (50 to 7300) $\mathrm{ml}$. The median length of hospital stay for HIPEC patients was 16 (7 to 104) days. One patient died 16 days following surgery and HIPEC. He presented with sepsis and multi-organ failure. The overall major (grade III to V) complication rate was of $39 \%$ (23 out of 58), including intra-abdominal abscesses $(22 \%, 13$ out of 58$)$, hemorrhage $(17 \%, 10$ out of 58$)$ and anastomotic leaks (10\% of patients, six out of $58 ; 17 \%$ of anastomoses, six out of 35). One patient in the HIPEC group experienced grade II neuropathy that lasted for one week following surgery. The same patient also developed grade III thrombocytopenia one week postoperatively.

Final pathology reports showed disseminated peritoneal adenomucinosis (DPAM) in 19 patients (24\%, 19 out of 78 ) (HIPEC $\mathrm{n}=14$, negative second-look (on initial pathology) $\mathrm{n}=5$, unresectable $\mathrm{n}=0$ ), PMCA-I in 41 patients $(53 \%, 41$ out of 78) (HIPEC $n=34$, negative second-look (on initial pathology) $n=3$, unresectable $n=4$ ) and PMCA in 18 patients $(23 \%, 18$ out of 78$)$ (HIPEC $n=10$, negative secondlook (on initial pathology) $n=1$, unresectable $n=7$ ).

\section{Survival rates}

The mean follow-up period was 33.7 months (median: 29.1; range: 2 to 100.8) for the entire series. The estimated five-year OS rate for the entire series was 66\% (95\% CI, 51 to 78 ). The estimated five-year OS was $100 \%$ for the negative second-look patients, $77 \%$ (95\% CI, 57 to 88 ) for the HIPEC patients, and 9\% (95\% CI, 1 to 33 ) for the unresectable patients $(P<0.0001)$ (Figure 1$)$. In the HIPEC group, at the time of data analysis, 15 patients $(25.9 \%)$ had isolated peritoneal recurrence, no patient had visceral recurrence only, and five patients (8.6\%) had both. The estimated five-year DFS for the HIPEC group was 50\% (95\% CI, 30 to 66 ) and $100 \%$ for the negative second-look group $(P=0.0478)$ (Figure 2).

On univariate analysis, histologic grade was an important prognostic indicator of both five-year DFS for HIPEC patients $(P=0.0016)$ and five-year OS for the entire cohort 


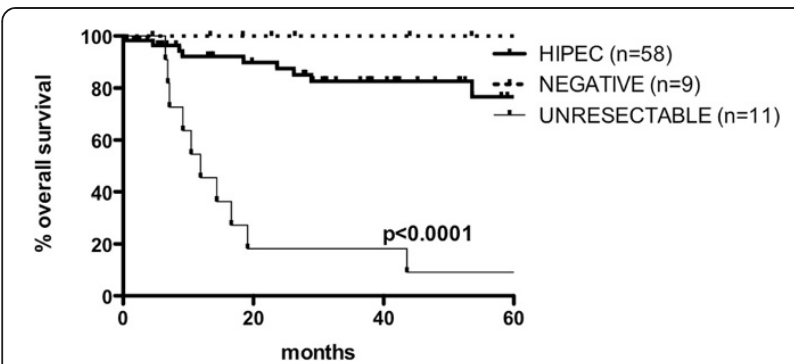

Figure 1 Overall survival at 60 months for patients who underwent complete surgical cytoreduction followed by hyperthermic intraperitoneal chemotherapy (HIPEC) who were found to have unresectable disease and those who had a negative second-look surgery.

$(P<0.00001)$ (Figure 3$)$. The respective five-year DFS and OS was $100 \%$ and $100 \%$ for DPAM, $40 \%$ (95\% CI, 17 to 62 ) and $40 \%$ (95\% CI, 17 to 62) for PMCA-I, as well as $20 \%$ (95\% CI, 1 to 55 ) and 20\% (95\% CI, 1 to 55) for PMCA. OS curves were statistically different between the PMCA-I and PMCA groups $(P<0.0003)$, between the DPAM and the PMCA groups $(P<0.0003)$ but they were not statistically significantly different between the DPAM and the PMCA-I groups. The cytoreduction score was also a significant prognostic factor of five-year DFS $(P=0.0172)$ with survival of $56 \%$ (95\% CI, 32 to 74 ) for CCR-0 and $24 \%$ (95\% CI, 2 to 61) for CCR-1, but a significant difference in OS was not found between the two groups, with a survival of $81 \%$ (95\% CI, 56 to 93 ) for CCR-0 and $64 \%$ (95\% CI, 32 to 85 ) for CCR-1. Other parameters such as age, sex, peritoneal index, duration of surgery, blood loss, systemic chemotherapy (neoadjuvant or adjuvant) and the occurrence of major complications had no significant influence on survival at univariate analysis.

Finally, the nine patients who underwent second-look laparotomy and were found to have no disease were also followed and are still disease-free.

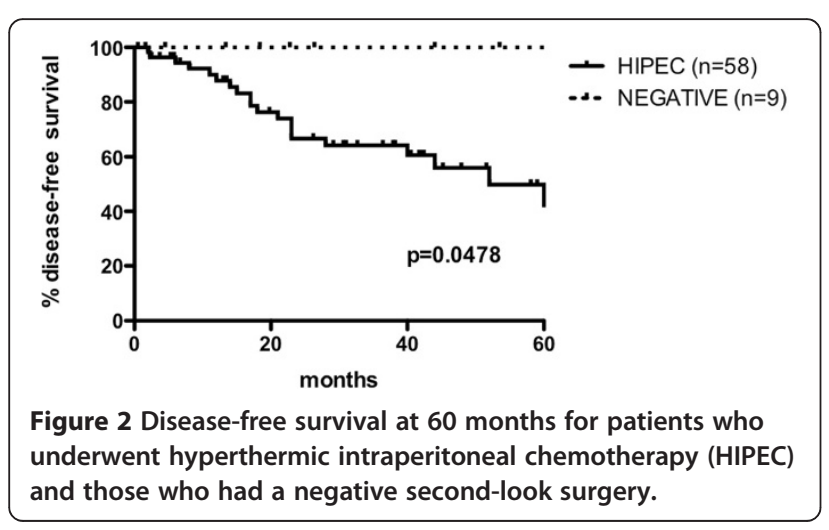

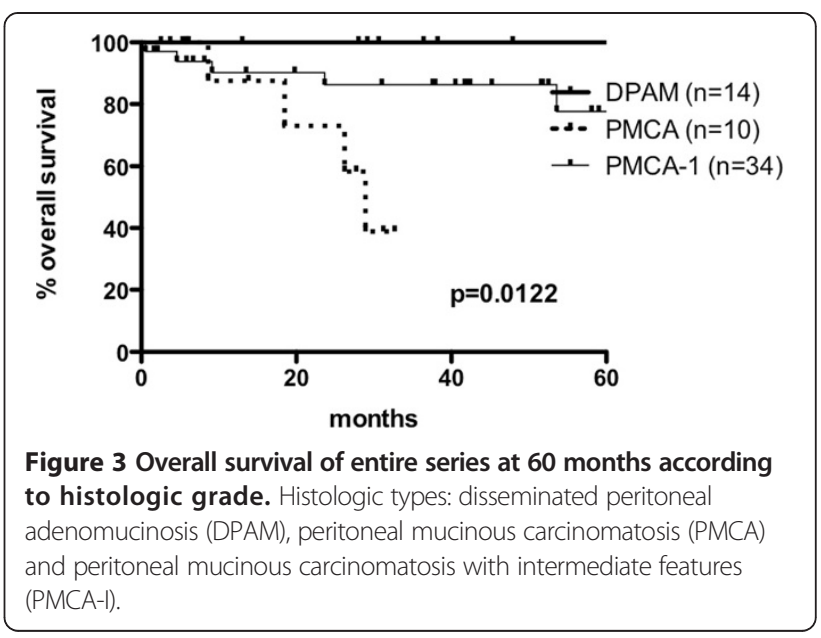

\section{Discussion}

$\mathrm{PC}$ arising from the appendix is a rare condition with a poor long-term prognosis. An aggressive surgical approach seems to be warranted and many centers have recently published their results. There seems to be a survival benefit of CRS and HIPEC administration compared to surgical debulking alone. For example, Gonzalez-Moreno and Sugarbaker $(\mathrm{n}=501)$, Elias et al. $(\mathrm{n}=301)$, Youssef et al. ( $\mathrm{n}=289)$ and Baratti et al. $(\mathrm{n}=104)$ reported the largest series of patients treated by CRS and HIPEC, with fiveyear OS rates of $71.9 \%, 73 \%, 87 \%$ and $71.9 \%$ respectively [5,10-12] (Table 1). These results compare favorably to the five-year OS rate of $53 \%$ by Gough et al. and $65 \%$ by Miner et al., who treated their patients with surgical debulking alone $[3,4]$.

Although effective, CRS is a lengthy procedure, with extensive dissection and numerous organ resections in order to achieve maximal cytoreduction (CCR-0 or CCR1) to allow for the administration of HIPEC. As already published, it was found that completeness of cytoreduction was a prognostic factor for DFS $[5,6,15]$. Although oxaliplatin has been demonstrated to penetrate the peritoneal tissues to a depth of up to $2.5 \mathrm{~mm}[17,25]$, it seems that CRS has a superior impact on prognosis than chemotherapy itself. Therefore, one should attempt maximal efforts to achieve complete macroscopic cytoreduction. However, the capacity to achieve CRS is dependent on the extent of peritoneal disease (PCI score) and grade of tumor $[8,13,14]$. In our series, a CCR-0 resection was achieved in 43 of the 68 patients (63\%), consistent with other teams $[6,8,10,11,15]$. Median PCI, at 13 , is somewhat lower than what was reported by other groups, raising some questioning regarding variability in PCI assessment among different teams.

In regards to tumor grading, there has been recent debate about Ronnett's original classification into three categories [26]. Indeed, many teams have lately been classifying PC arising from the appendix in two categories (low- versus 
Table 1 Effectiveness of perioperative intraperitoneal chemotherapy (PIC) for peritoneal carcinomatosis arising from the appendix (pseudomyxoma peritonei)

\begin{tabular}{|c|c|c|c|c|c|c|c|c|c|c|}
\hline \multirow{2}{*}{$\begin{array}{c}\text { Chief- } \\
\text { Investigator }\end{array}$} & \multirow[t]{2}{*}{ PIC } & \multirow[t]{2}{*}{$\mathrm{n}$} & \multirow{2}{*}{$\begin{array}{l}\text { Median } \\
\text { follow-up } \\
\text { (months) }\end{array}$} & \multicolumn{4}{|c|}{ Overall survival (\%) } & \multicolumn{3}{|c|}{ Disease-free survival (\%) } \\
\hline & & & & 1 year & 3 years & 5 years & 10 years & 1 year & 3 years & 5 years \\
\hline Sugarbaker [5] & HIPEC or EPIC (MMC) & 501 & 48 & --- & --- & 71.9 & 54.5 & -- & --- & --- \\
\hline Elias [11] & HIPEC (OX or MMC) & 301 & 88 & --- & --- & 73 & --- & --- & --- & 56 \\
\hline Moran [12] & HIPEC (MMC) & 289 & 39 & --- & -- & 87 & 74 & --- & --- & 70 \\
\hline Choudry [15] & HIPEC (MMC) & 282 & 24 & --- & 67.4 & 52.7 & --- & --- & 45.1 & 32.1 \\
\hline Morris [6] & HIPEC (MMC) & 106 & 23 & --- & -- & 75 & -- & 71 & 51 & 38 \\
\hline Deraco [10] & HIPEC (MMC) & 104 & 37 & --- & --- & 71.9 & --- & -- & --- & 38.8 \\
\hline Zoetmulder [8] & HIPEC (MMC) & 103 & 51.5 & 90 & 70.9 & 59.5 & $>50$ & --- & 43.6 & 37.4 \\
\hline Sardi (PMCA) [14] & HIPEC (MMC) & 77 & 18 & 88 & 56 & 40 & --- & --- & --- & --- \\
\hline Temple [13] & HIPEC (MMC) & 58 & 28 & --- & 76 & 62 & --- & --- & 48 & 42 \\
\hline Current study & HIPEC (OX) & 58 & 29.1 & --- & --- & 66.2 & --- & --- & --- & 50 \\
\hline
\end{tabular}

EPIC, early postoperative intraperitoneal chemotherapy; HIPEC, Hyperthermic intraperitoneal chemotherapy; MMC, mitomycin C; OX, oxaliplatin.

high-grade) by regrouping PMCA-I lesions with DPAMs because of findings of comparable survival [15,27]. Our findings that the OS of PMCA-I patients is closer to DPAM than PMCA on univariate analysis support that classification. Others proposed that $\mathrm{CP}$ arising from non-mucinous adenocarcinoma (classic type) be regarded as a separate category because they lead to the poorest outcome [28]. Although a binary classification seems to be simpler and more reproducible, until a consensus is established, we have chosen to use the original Ronnett's classification, in order to compare with other groups and to allow for continuity with the preliminary results we published in 2008 [21].

CRS plus HIPEC remains a procedure with a high rate of major complications (Table 2) $[6,8,10-12,14,15,29]$. However, it is noteworthy that the frequency of systemic (hematologic) toxicity was low, confirming the reports of Elias et al. [20]. The fact that chemoperfusion takes $60 \mathrm{mi}-$ nutes less to perform than for the mitomycin-C is another reason why we prefer to use oxaliplatin for HIPEC.

A total of 15 patients (26\%) in the HIPEC group presented an isolated peritoneal recurrence, a median of
18 months after the HIPEC procedure. Five of them were eligible for repeat surgery and accepted to undergo another CRS with HIPEC procedure using mitomycin-C.

It is important to mention that one patient with PMCA disease in the negative second-look surgery in our preliminary series finally received CRS and HIPEC after presenting a peritoneal recurrence 36 months after a negative exploration. He presented with another peritoneal recurrence a year after the HIPEC with oxaliplatin. He then underwent another CRS and HIPEC with mitomycin-C but succumbed to the disease 16 months later. With a median follow-up period of more than two years, the OS and DFS survival for the remaining nine negative second-look patients are both $100 \%$, which gives us cause to believe that a prophylactic HIPEC may not be warranted for patients with low-grade disease.

Elias et al. recently published the results of their retrospective trial of prophylactic HIPEC for asymptomatic patients at high risk of presenting colorectal PC (resected synchronous macroscopic PC, ovarian metastasis or tumor perforation) [30]. Peritoneal disease was found in more

Table 2 Morbidity and mortality of cytoreductive surgery combined with perioperative intraperitoneal chemotherapy (PIC) in peritoneal carcinomatosis arising from the appendix (pseudomyxoma peritonei)

\begin{tabular}{|c|c|c|c|c|c|}
\hline Chief-Investigator & Center & $\mathrm{n}$ & Morbidity (\%) & Hematologic toxicity (\%) & Mortality (\%) \\
\hline Elias [11] & Multicentric & 301 & 40 & 20 & 4.4 \\
\hline Morran [12] & Basingstoke, UK & 289 & 7 & 2 & 1.4 \\
\hline Choudry [15] & Pittsburgh & 282 & 24.8 & --- & 1.1 \\
\hline Sugarbaker [29] & Washington, DC & 155 & 27 & --- & 2 \\
\hline Sardi [14] & Baltimore & 77 & 27 & --- & 0 \\
\hline Morris [6] & Sydney & 106 & 49 & --- & 3 \\
\hline Deraco [10] & Milan & 104 & 25 & 3.8 & 1 \\
\hline Zoetmulder [8] & Amsterdam & 103 & 54 & 10.7 & 11 \\
\hline Current study & Montreal & 58 & 40 & 1.7 & 1.7 \\
\hline
\end{tabular}


than half of patients despite negative imaging. All patients (including those without macroscopic evidence of peritoneal disease recurrence) underwent HIPEC with oxaliplatin. OS at five years is encouraging, at $90 \%$. We believe that patients with appendiceal carcinomatosis with high-grade disease (PMCA, classic adenocarcinoma or signet-ring cell lesions) would benefit from a similar approach and receive prophylactic HIPEC.

Concerning patients with unresectable disease, they had a more aggressive disease (64\% PMCA and 36\% PMCA-I) and 10 out of the 11 patients died a median of 11.2 months after surgery. In a study of 18 patients with peritoneal carcinomatosis arising from an appendiceal adenocarcinoma with signet-ring cells, 10 patients were able to get CRS and HIPEC, whereas eight patients were only administered intravenous chemotherapy because of failure to get complete CRS. Median survival was found to be 27 versus 15 months, respectively [31]. Although not statistically significant $(P=0.12$ ), it seems that the longer survival supports aggressive surgical management, while being reasonable in order to prevent unwarranted complications. In our series, two patients with bulky disease (PMCA-I) that was deemed unresectable at first laparotomy received intravenous systemic chemotherapy (FOLFOX) followed by abdominal reexploration six months later. They then underwent a CCR-0 cytoreduction and HIPEC. With a follow-up period of 42 and 45 months respectively, they are both alive without evidence of disease.

\section{Conclusions}

This therapeutic approach seems both feasible and safe in selected patients. Survival and morbidity with oxaliplatin is similar to what has been reported for HIPEC with mitomycin-C. The major complication rates of these procedures remain high and constant efforts to reduce the operative morbidity is warranted. Recurrence is, however, frequent and represents a challenge. Chemotherapy is warranted for patients with PC deemed unresectable upon initial exploration and a second-look should be performed since chemotherapy can reduce tumor burden enough to allow for complete cytoreduction and HIPEC.

\section{Abbreviations}

CTCAE: Common Terminology Criteria for Adverse Events; CRS: complete surgical cytoreduction; CCR: completeness of cytoreduction; DFS: disease-free survival; DPAM: disseminated peritoneal adenomucinosis; EPIC: early postoperative intraperitoneal chemotherapy; HIPEC: hyperthermic intraperitoneal chemotherapy; ICU: intensive care unit; MMC: mitomycin C; OS: overall survival; OX: oxaliplatin; PIC: perioperative intraperitoneal chemotherapy; PCl: Peritoneal Cancer Index; PC: peritoneal carcinomatosis; PMCA: peritoneal mucinous carcinomatosis; PMCA-I: peritoneal mucinous carcinomatosis with intermediate features.
}

\section{Competing interests}

The authors declare that they have no competing interests.

\section{Authors' contributions}

EM, LS, PDu, PDr conceptualized and designed the study. AM, SF, GL and YEL reviewed the study design. EM acquired the data and prepared the manuscript. EM, PDr, and LS analyzed and interpreted the data. PDu, LS and AM edited the manuscript. All authors read and approved the final manuscript.

\section{Author details}

'Department of Surgery, Hôpital Maisonneuve-Rosemont, University of Montreal, 5415 boulevard de l'Assomption, Montréal, Québec H1T 2 M4, Canada. ${ }^{2}$ Department of Anesthesiology, Hôpital Maisonneuve-Rosemont, University of Montreal, 5415 boulevard de l'Assomption, Montréal, Québec H1T 2 M4, Canada. ${ }^{3}$ Department of Pathology, Hôpital

Maisonneuve-Rosemont, University of Montreal, 5415 boulevard de I'Assomption, Montréal, Québec H1T 2 M4, Canada. ${ }^{4}$ Department of Pharmacy, Hôpital Maisonneuve-Rosemont, University of Montreal, 5415 boulevard de l'Assomption, Montréal, Québec H1T 2 M4, Canada.

Received: 3 March 2013 Accepted: 20 October 2014

Published: 7 November 2014

\section{References}

1. McCusker ME, Cote TR, Clegg LX, Sobin LH: Primary malignant neoplasms of the appendix: a population-based study from the surveillance, epidemiology and end-results program, 1973-1998. Cancer 2002, 94:3307-3312

2. Connor SJ, Hanna GB, Frizelle FA: Appendiceal tumors: retrospective clinicopathologic analysis of appendiceal tumors from 7,970 appendectomies. Dis Colon Rectum 1998, 41:75-80.

3. Gough DB, Donohue JH, Schutt AJ, Gonchoroff N, Goellner JR, Wilson TO, Naessens JM, O'Brien PC, van Heerden JA: Pseudomyxoma peritonei. Long-term patient survival with an aggressive regional approach. Ann Surg 1994, 219:112-119.

4. Miner TJ, Shia J, Jaques DP, Klimstra DS, Brennan MF, Coit DG: Long-term survival following treatment of pseudomyxoma peritonei: an analysis of surgical therapy. Ann Surg 2005, 241:300-308.

5. Gonzalez-Moreno S, Sugarbaker PH: Right hemicolectomy does not confer a survival advantage in patients with mucinous carcinoma of the appendix and peritoneal seeding. Br J Surg 2004, 91:304-311.

6. Chua TC, Yan TD, Smigielski ME, Zhu KJ, Ng KM, Zhao J, Morris DL: Long-term survival in patients with pseudomyxoma peritonei treated with cytoreductive surgery and perioperative intraperitoneal chemotherapy: 10 years of experience from a single institution. Ann Surg Oncol 2009, 16:1903-1911.

7. Guner Z, Schmidt U, Dahlke MH, Schlitt HJ, Klempnauer J, Piso P: Cytoreductive surgery and intraperitoneal chemotherapy for pseudomyxoma peritonei. Int I Colorectal Dis 2005, 20:155-160

8. Smeenk RM, Verwaal VJ, Antonini N, Zoetmulder FA: Survival analysis of pseudomyxoma peritonei patients treated by cytoreductive surgery and hyperthermic intraperitoneal chemotherapy. Ann Surg 2007, 245:104-109.

9. Stewart JHT, Shen P, Russell GB, Bradley RF, Hundley JC, Loggie BL, Geisinger KR, Levine EA: Appendiceal neoplasms with peritoneal dissemination: outcomes after cytoreductive surgery and intraperitoneal hyperthermic chemotherapy. Ann Surg Oncol 2006, 13:624-634.

10. Baratti D, Kusamura S, Nonaka D, Langer M, Andreola S, Favaro M, Gavazzi C, Laterza B, Deraco M: Pseudomyxoma peritonei: clinical pathological and biological prognostic factors in patients treated with cytoreductive surgery and hyperthermic intraperitoneal chemotherapy (HIPEC). Ann Surg Oncol 2008, 15:526-534.

11. Elias D, Gilly F, Quenet F, Bereder JM, Sideris L, Mansvelt B, Lorimier G, Glehen O: Pseudomyxoma peritonei: a French multicentric study of 301 patients treated with cytoreductive surgery and intraperitoneal chemotherapy. Eur J Surg Oncol 2010, 36:456-462.

12. Youssef H, Newman C, Chandrakumaran K, Mohamed F, Cecil TD, Moran BJ: Operative findings, early complications, and long-term survival in 456 patients with pseudomyxoma peritonei syndrome of appendiceal origin. Dis Colon Rectum 2011, 54:293-299.

13. Hamilton $T$, Lanuke $K$, Mack LA, Temple WJ: Long-term follow-up in the treatment of peritoneal carcinomatosis. Am J Surg 2011, 201:650-654.

14. El Halabi H, Gushchin V, Francis J, Athas N, Macdonald R, Nieroda C, Studeman K, Sardi A: The role of cytoreductive surgery and heated intraperitoneal chemotherapy (CRS/HIPEC) in patients with high-grade 
appendiceal carcinoma and extensive peritoneal carcinomatosis. Ann Surg Oncol 2012, 19:110-114.

15. Austin F, Mavanur A, Sathaiah M, Steel J, Lenzner D, Ramalingam $L_{\text {, }}$ Holtzman M, Ahrendt S, Pingpank J, Zeh HJ, Bartlett DL, Choudry HA: Aggressive management of peritoneal carcinomatosis from mucinous appendiceal neoplasms. Ann Surg Oncol 2012, 19:1386-1393.

16. Sugarbaker PH: Laboratory and clinical basis for hyperthermia as a component of intracavitary chemotherapy. Int J Hyperthermia 2007, 23:431-442

17. Los G, Smals OA, van Vugt MJ, van der Vlist M, den Engelse L, McVie JG, Pinedo HM: A rationale for carboplatin treatment and abdominal hyperthermia in cancers restricted to the peritoneal cavity. Cancer Res 1992, 52:1252-1258

18. Piche N, Leblond FA, Sideris L, Pichette V, Drolet P, Fortier LP, Mitchell A, Dube $P$ : Rationale for heating oxaliplatin for the intraperitoneal treatment of peritoneal carcinomatosis: a study of the effect of heat on intraperitoneal oxaliplatin using a murine model. Ann Surg 2011, 254:138-144.

19. Elias D, Laurent S, Antoun S, Duvillard P, Ducreux M, Pocard M, Lasser P: [Pseudomyxoma peritonei treated with complete resection and immediate intraperitoneal chemotherapy]. Gastroenterol Clin Biol 2003, 27:407-412.

20. Elias D, Bonnay M, Puizillou JM, Antoun S, Demirdjian S, El OA, Pignon JP, Drouard-Troalen L, Ouellet JF, Ducreux M: Heated intra-operative intraperitoneal oxaliplatin after complete resection of peritoneal carcinomatosis: pharmacokinetics and tissue distribution. Ann Oncol 2002, 13:267-272

21. Marcotte E, Sideris L, Drolet P, Mitchell A, Frenette S, Leblanc G, Leclerc YE, Dube P: Hyperthermic intraperitoneal chemotherapy with oxaliplatin for peritoneal carcinomatosis arising from appendix: preliminary results of a survival analysis. Ann Surg Oncol 2008, 15:2701-2708.

22. Dindo D, Demartines N, Clavien PA: Classification of surgical complications: a new proposal with evaluation in a cohort of 6336 patients and results of a survey. Ann Surg 2004, 240:205-213.

23. Ronnett BM, Yan H, Kurman RJ, Shmookler BM, Wu L, Sugarbaker PH: Patients with pseudomyxoma peritonei associated with disseminated peritoneal adenomucinosis have a significantly more favorable prognosis than patients with peritoneal mucinous carcinomatosis. Cancer 2001, 92:85-91.

24. Sugarbaker PH: Intraperitoneal chemotherapy and cytoreductive surgery for the prevention and treatment of peritoneal carcinomatosis and sarcomatosis. Semin Surg Oncol 1998, 14:254-261.

25. Jacquet $\mathrm{P}$, Sugarbaker $\mathrm{PH}$ : Clinical research methodologies in diagnosis and staging of patients with peritoneal carcinomatosis. Cancer Treat Res 1996, 82:359-374.

26. Ronnett BM, Zahn CM, Kurman RJ, Kass ME, Sugarbaker PH, Shmookler BM: Disseminated peritoneal adenomucinosis and peritoneal mucinous carcinomatosis. A clinicopathologic analysis of 109 cases with emphasis on distinguishing pathologic features, site of origin, prognosis, and relationship to "pseudomyxoma peritonei". Am J Surg Pathol 1995 19:1390-1408.

27. Bradley RF, Stewart JHT, Russell GB, Levine EA, Geisinger KR: Pseudomyxoma peritonei of appendiceal origin: a clinicopathologic analysis of 101 patients uniformly treated at a single institution, with literature review. Am J Surg Pathol 2006, 30:551-559.

28. Bruin SC, Verwaal VJ, Vincent A, van't Veer LJ, van Velthuysen ML: A clinicopathologic analysis of peritoneal metastases of colorectal and appendiceal origin. Ann Surg Oncol 2010, 17:2330-2340.

29. Sugarbaker PH, Alderman R, Edwards G, Marquardt CE, Gushchin V, Esquivel J, Chang D: Prospective morbidity and mortality assessment of cytoreductive surgery plus perioperative intraperitoneal chemotherapy to treat peritoneal dissemination of appendiceal mucinous malignancy. Ann Surg Oncol 2006, 13:635-644
30. Elias D, Honore C, Dumont F, Ducreux M, Boige V, Malka D, Burtin P, Dromain C, Goere D: Results of systematic second-look surgery plus HIPEC in asymptomatic patients presenting a high risk of developing colorectal peritoneal carcinomatosis. Ann Surg 2011, 254:289-293.

31. Chua TC, Pelz JO, Kerscher A, Morris DL, Esquivel J: Critical analysis of 33 patients with peritoneal carcinomatosis secondary to colorectal and appendiceal signet ring cell carcinoma. Ann Surg Oncol 2009, 16:2765-2770.

doi:10.1186/1477-7819-12-332

Cite this article as: Marcotte et al.: Hyperthermic intraperitoneal chemotherapy with oxaliplatin as treatment for peritoneal carcinomatosis arising from the appendix and pseudomyxoma peritonei: a survival analysis. World Journal of Surgical Oncology 2014 12:332.

\section{Submit your next manuscript to BioMed Central and take full advantage of:}

- Convenient online submission

- Thorough peer review

- No space constraints or color figure charges

- Immediate publication on acceptance

- Inclusion in PubMed, CAS, Scopus and Google Scholar

- Research which is freely available for redistribution

Submit your manuscript at www.biomedcentral.com/submit
C BioMed Central 